\title{
Sexual activity and the risk of acute uncomplicated urinary tract infection in premenopausal women: implicationsfor reproductive health programming
}

\begin{abstract}
Acute uncomplicated UTIs occur in otherwise healthy individuals with no structural or functional urinary tract abnormalities. Young sexually active women are at highest risk thereof.

Its pathogenesis is complex, influenced by the host's biological and behavioural factors, as well as virulence properties of the infecting micro-organisms. The main source of the micro-organisms is the individual's own faecal flora. E. coli is the commonest bacteria accounting for $75-90 \%$ of the isolates. The major risk factors include frequency of and recent sexual intercourse, use of contraceptive diaphragm, male condoms and spermicides and use of some antimicrobials.

Young women are highly sexually active with a potential of variety of partners, and variety of sexual activities such heterosexual anal intercourse, which increase the risk of UTI. Sexual and reproductive health programmes stress the importance of safer sex including use of barrier contraceptive methods to prevent unwanted pregnancy, HIV infection and STIs. This has resulted in significant uptake of spermicide-coated condoms amongst young people globally.

The association of acute uncomplicated UTI with sexual activities including vaginal and heterosexual anal intercourse (HAI), and use of spermicide-coated condoms may persuade some to stop using condoms, thus negatively impacting on the gains made in reproductive health programmes. There is need for a paradigm shift in international and national reproductive health programming, as well as public education on sexual and reproductive health as well as risk factors for uncomplicated UTI and preventive strategies. There is also need for locally relevant operations research to better understand the risk factors, among other issues so as to inform health service programming and delivery, including preventive strategies.
\end{abstract}

Volume 9 Issue I - 2018

Lema VM,' Lema APV²

'Professor of Obstetrics \& Gynaecology, Aga Khan University, Kenya

${ }^{2}$ Medical Officer, Nairobi Hospital, Kenya

Correspondence: Lema VM, Professor of Obstetrics \& Gynaecology, Honorary Faculty, Aga Khan University, Nairobi, Kenya,Tel 254-7II860582,

Emailvmlema@gmail.com,sreprohealth@gmail.com

Received: July 30, 2017| Published: February 08, 2018
Abbreviations: HAI, Heterosexual Anal Intercourse; UTI, Urinary Tract Infection; UPEC, Uropathogenic E. Coli

\section{Introduction}

Urinary tract infection (UTI) is defined as the invasion and multiplication of non-resident pathogenic micro-organisms into the urinary tract, resulting in an inflammatory response in the urothelium, which progresses to overt disease. Urinary tract infections (UTIs) are considered one of the most common bacterial infections, and by some authorities the most common globally, accounting for a significant proportion of medical consultations in primary as well as outpatient settings. ${ }^{1,2}$

The actual magnitude of UTI is unknown and is difficult to establish because it is not a notifiable health condition, some patients opt to self-diagnose and self-medicate and quite often in outpatient settings uncomplicated UTI is treated symptomatically without microbiological evidence thereof. The differences in reported incidences/prevalence rates are influenced by the criteria used for its diagnosis and specimen-collection technique among other factors. Hooton et $\mathrm{al}^{3}{ }^{3}$ in their study on young people in the USA, estimated the incidence of UTI at 0.5 to 0.7 per person per year. Other estimates indicate that UTI accounts for $\geq 8.6$ million physician visits and $\geq 1.0$ million hospital admissions each year in the USA. ${ }^{4}$ In a populationbased study in Canada, Nicolle et al. ${ }^{5}$ reported that the overall rate of hospitalisation for pyelonephritis in women was about 1:1000 population, which was considered an underestimate cognisant of the fact that not all patients with acute pyelonephritis get admitted or require hospitalization. ${ }^{6}$ This did not include acute cystitis, which is more common than pyelonephritis.

According to the new classification of urinary tract infection, acute uncomplicated urinary tract infection (UTI) includes sporadic and recurrent community-acquired episodes of acute cystitis and acute pyelonephritis, in otherwise healthy individuals with no known risk factors, with risk of recurrences but no risk of more severe outcome, and partly extra-urogenital risk factors, based on the ORENUC system. ${ }^{7,8}$ Young healthy, sexually active women are particularly at risk of acute uncomplicated (or community-acquired) urinary tract infections. Community-acquired UTI is infrequent before puberty and/or sexual debut. About a third of women will have UTI requiring antimicrobial therapy by age $24,, 1040-50 \%$ of women will experience at least one episode in their lifetime ${ }^{11,12}$ with a quarter or more having a second and $20-50 \%$ of them having recurrent UTI, defined as $\geq 3$ laboratory confirmed episodes of UTI over a twelve months period..$^{13}$

Although acute uncomplicated urinary tract infection is not accompanied by long term sequelae, it is associated with significant morbidity and health costs, because of its magnitude, the symptomatology and recurrences.

This paper discusses the pathogenesis of uncomplicated UTI in premenopausal women, with specific reference to the major 
risk factors, i.e. vaginal intercourse and other sexual behaviours/ activities and implications thereof to sexual and reproductive health programming and service delivery.

\section{Pathogenesis}

Pathogenesis of acute uncomplicated UTI is reportedly complex. It is influenced by many biological (i.e. structural and functional characteristics of the genitourinary and alimentary systems) and behavioural factors of the host, i.e. the individual woman; as well as virulence properties of the infecting microorganisms..$^{14,15}$ For an infection to occur in any part of the human body, the pathogenic microorganisms must gain entry into the body, organ or tissue in question, survive therein, multiply and invade the respective tissues, because an inflammatory response which then presents with related clinical symptoms. Most body tissues, such as the urinary tract or the vagina, have innate and adaptive immune mechanisms which protect them against invasion by, survival and multiplication of non-resident microorganisms.

As opposed to complicated UTI, acute uncomplicated UTI is not associated with any underlying structural or functional abnormalities in the urinary tract. It is infrequent in young girls and early adolescence, its incidence increasing dramatically following sexual debut, often in late adolescence and/or early 20 s, remaining high throughout the woman's adult life. ${ }^{16}$

The primary route in the acquisition of uncomplicated UTI in women is by ascendance of the microorganisms. ${ }^{17}$ Haematogenous and lymphatic spread of uropathogens to the urinary tract is either rare or unknown. ${ }^{18}$ Most of the uropathogens in community-acquired UTI in young sexually active women originate from the rectal/enteric flora. ${ }^{14,18}$ The possibility of sexual transmission of uropathogenic $E$. coli (UPEC) from a partner has been suggested, ${ }^{19}$ but this is perhaps very rare. Vaginal colonisation by the uropathogens is considered a pre-requisite to and an initial step in the pathogenesis of communityacquired UTI in young women. This is accompanied by colonisation of the peri-urethral area and lower third of the urethra from where the uropathogens can enter the bladder during sexual intercourse or urethral manipulation. ${ }^{14}$

\section{The uropathogens}

Uropathogenic E. coli (UPEC) is the commonest microbial agent in acute uncomplicated UTI, responsible for $75-90 \%$ of infections, ${ }^{20-22}$ followed by Staphylococcus saprophyticus which accounts for 5-15\% of infections. ${ }^{3,23}$ Other bacteria include Proteus spp, Klebsiella spp, Pseudomonas spp, Citrobacter, Enterobacter, Enterococcus faecalis which are an infrequent cause of community-acquired UTI in young sexually active women. ${ }^{11,24}$ Certain virulence determinants of uropathogens are said to confer a selective advantage to the strains possessing them which enable the microorganisms to colonise and infect the urinary tract. ${ }^{20,25}$ Uropathogens' virulence determinants or properties are considered more important in the normal host than those with urinary tract abnormalities. ${ }^{14}$

E. coli being the commonest bacteria causing UTI has been studied most with regards to its pathogenicity and virulence than the others. E. coli is a commensal of the large intestine where it is considered beneficial. However some of its strains deviate from their commensal status and assume a pathogenic course capable of infecting tissues and causing diseases in the same body, such as UTI. ${ }^{26,27}$ There is also evidence to suggest that UPEC originates from eating poultry contaminated with avian E. coli (APEC) ${ }^{28}$ UPEC possess an enhanced ability to cause infection outside the intestinal tract such as
UTI by changing from its harmless nature in the nutrient-rich intestine to a virulent pathogen in a nutrition-depleted environment such as the urinary tract. . $^{27,29,30}$

UPEC is able to utilise diverse carbon sources for its energy, ${ }^{30,31}$ and is capable of evading or surviving the effects of neutrophils during UTI. Its specific virulence factors i.e. adhesive organelles called fimbriae or pilli, allow it to bind and invade host cells and tissues in the urinary tract. ${ }^{13}$ Some E.coli strains express an increase in $\mathrm{K}$-antigen production which confers protection to the microorganism from leukocyte phagocytosis, thus enabling UPEC to survive and multiply in the urinary tract. ${ }^{14}$ Expression of iron-chelating factors which enable it to pilfer iron stores, depleting available iron from urothelium, deployment of an array of toxins such as haemolysin and cytotoxic necrotising factor 1 which not only facilitate invasion but also release of host nutrients and disable the immune effector cells have been suggested. ${ }^{20,32,33}$

S. saprophyticus, the second most common bacteria causing community-acquired UTI has also being shown to possess several virulence properties such as haemagglutination, adherence to human uroepithelial cells, and production of extracellular enzymes such as urease, which inhibit growth of bacteria. ${ }^{34}$

\section{Host factors}

These include anatomical (structural), biological (functional) features of the female genito-urinary and alimentary systems and behavioural characteristics of the individual woman.

Anatomical: The anatomical arrangement of the female external genitalia and anus is important in the pathogenesis of communityacquired urinary tract infection in young women, and it explains why they are at greater risk thereof than men. The female urethral meatus is within the vestibule, enclosed by the labia majora and minora, an environment which is most of the time wet or moist. It is in close proximity to the vaginal introitus which exposes it to a risk of trauma as well as bacterial contamination during sexual intercourse. The vaginal introitus and urethral meatus are in close proximity to the anus, making it easy for enteric bacteria to gain access into the vagina and urethra. The female urethra in quite short, measuring approximately $4 \mathrm{~cm}$ long and is adjacent to the vagina a factor which enhances the massaging effect of the urethra during sexual intercourse, pushing uropathogens into the bladder. The distance between the urethra and anus was shown to be more important, in that the shorter the distance the higher was the risk of UTI in one study. ${ }^{35}$

Biological properties:Alterations in the normal vaginal flora is critical in facilitating vaginal colonisation with pathogenic organisms such as coliforms, setting the stage for development of UTI. Loss of the hydrogen peroxide-producing lactobacilli which is protective may predispose a woman to vaginal colonisation by uropathogens. ${ }^{22}$

The urinary system has its protective mechanisms against infection. These include the high urine flow rate, voiding frequency, urine osmolality, its $\mathrm{pH}$ and organic acids; bladder mucopolysaccharides, secretory $\operatorname{IgA}$ and other proteins secreted by the uroepithelial cells. Factors that interfere with or interrupt these protective mechanisms facilitate bacterial colonisation of the vagina, entry into the urethra and bladder, its multiplication and survival therein leading to infection and symptoms related thereto. ${ }^{36}$

\section{Behavioural factors}

Sexual intercourse: UTI in infrequent in girls and young adolescents, but increases dramatically following sexual debut. ${ }^{9,37,38}$ Some of the 
earliest studies on the association between sexual intercourse and community-acquired UTI showed that UTI in young women was preceded by sexual intercourse and most of those with UTI had used the female on-top sexual position. ${ }^{39}$ Foxman et al. ${ }^{37}$ reported that UTI increased from $12.5 \%$ in age group $14-17$ to $37.8 \%$ in those aged 18 24

Penile-vaginal intercourse has been shown to be a major risk factor for community-acquired UTI in premenopausal women, in particular its frequency and recent activity thereof. ${ }^{14,15,37,40}$ The relative odds of acute cystitis during the first 48 hours after sexual intercourse increase by a factor as great as $60 .{ }^{41}$ Nicolle et al..$^{42}$ showed that about $80 \%$ of UTI in young college girls occurred within 24 hours after sexual intercourse. Hooton et al. ${ }^{3}$ reported that sexual intercourse on three days in a week increased the risk by 2.6 times vs those who never had sexual intercourse, and if it was daily the risk increased ninefold. Studies have not shown an association between the number of partners and risk of sporadic acute uncomplicated UTI. In one study, women with two or fewer sexual partners in the preceding year $(n=793)$ were compared with those with three or more partners $(\mathrm{n}=204)$, and it showed that UTI was not significantly more common in those with three or more partners $(\mathrm{x} 2=0.036 ; \mathrm{p}>0.05)$. However significant bacteriuria was detected most often $(11.2 \%)$ in women who had had sexual intercourse within the preceding 24 hours. ${ }^{43}$

Vaginal sexual intercourse is thought to increase the risk of UTI through bacterial contamination of the vaginal introitus and periurethral area and introduction of uropathogens into the urethra and bladder by massaging the urethra and possibly trauma to the urethral meatus. ${ }^{3,14,15}$

Vaginal acquisition of uropathogens from a woman's infected male partner/spouse has been reported, but is considered a rare cause of uncomplicated UTI. ${ }^{14} \mathrm{Al}$-Walli et al. ${ }^{44}$ reported a case of recurrent UTI in a young woman whose husband had chronic prostatitis.

Other sexual activities are also associated with an increased risk of UTI. In their study, Foxman et al. ${ }^{37}$ noted that all types of sexual behaviour/activities were associated with increased risk of UTI, i.e. vaginal intercourse alone, or in combination with digital stimulation and or receptive oral sex. Heterosexual receptive anal intercourse (HAI) has been reported to confer an increased risk of uncomplicated UTI in premenopausal women. ${ }^{45,46}$ Lema (2015) published casereports of young women with severe uncomplicated UTI in Nairobi Kenya. They had vaginal intercourse following anal intercourse, without protection, within the preceding seven days and the men did not wash their penises in between anal and vaginal intercourse. ${ }^{46}$ Following these reported cases the author has attended to a few more young sexually active women with similar stories in his private clinic, in Nairobi Kenya. Handley et al. ${ }^{24}$ opined that anal intercourse may facilitate transfer of uropathogens from the female anus into the vaginal vestibule and urethra leading to UTI, after which vaginal intercourse may facilitate their entry into the urethra and bladder.

Contraceptives: Use of diaphragm with or without spermicides, male condoms with or without spermicides and spermicides themselves, has been shown to significantly increase the risk of UTI in young women..$^{14,15,37}$ In their study on the association of diaphragm and first UTI Foxman and Frerichs (1985) showed that sexually active women with primary UTI who had intercourse three or more times per week and used the diaphragm had at least a 63 per cent chance that their primary UTI was attributable to use of diaphragm..$^{47}$ The diaphragm is thought to cause urinary stasis because of its positioning in the vagina among other mechanisms.
Foxman et al. $^{48}$ in their study on first time UTI among female university students, noted that using lubricated condoms increased the odds ratio of UTI by $29 \%(95 \% \mathrm{CI}=3.1-1,335)$, and that spermicide cream/gel with unlubricated condoms was associated with a 2 to 8 fold increased risk of first time UTI. In an earlier study, Foxman et al., ${ }^{37}$ had shown that vaginal intercourse with male condoms increased the risk of UTI by $43 \%(\mathrm{OR}=1.43,95 \% \mathrm{CI}=1.09-1.89)$. Handley et al. ${ }^{40}$ showed that use of male condoms was associated with an increased UTI risk and that the largest risk was associated with exclusive condom use and use of Nonoxynol-9 coated condoms. The male condom increases the risk of acquiring UTI in the woman through an allergic reaction to the latex rubber or the lubricant, and mechanical trauma of sexual intercourse. ${ }^{49}$

Spermicides on their own have been shown to increase the odds of uncomplicated UTI by E. coli or by S. saprophyticus by a factor of 2 to 3, irrespective of whether a diaphragm or spermicide-coated condoms were used concurrently.,49 Spermicides have been shown to increase the risk of UTI independent of sexual intercourse, ${ }^{3}$ with a higher risk the more frequent it is used ${ }^{49}$ Spermicides are thought to increase the risk of UTI by altering the vaginal microflora, i.e. depleting the concentration of lactobacilli, and by increasing vaginal colonization. ${ }^{49,50}$ A study by Rosenstein et al.$^{50}$ showed that there was an increase in vaginal coliforms and a decrease in vaginal lactobacilli after Nonoxynol-9 instillation into the vagina in the absence of sexual intercourse or diaphragm use. Nonoxynol-9 suppresses growth of lactobacilli in the vagina as well as enhance bacteria adherence to the vaginal epithelial cells. ${ }^{50,51}$ However not all studies have shown significant effects of Nonoxynol-9 on hydrogen-producing lactobacilli.

Antimicrobials: The use of certain antimicrobial agents has been shown to increase the risk of uncomplicated UTI in young women by adversely affecting vaginal microflora. ${ }^{52,53}$ Smith et al..$^{53}$ in their prospective study on premenopausal college women showed they were at increased risk of UTI if they had used antimicrobial agents in the previous 15-28 days (i.e. three to four weeks) but not in the previous $\leq 14$ days. The explanation was that in the fisrt two weeks the antimicrobial effects were possibly still in effect but would have worn off by the end of that period.

\section{Recurrent uncomplicated urinary tract infections}

This has hitherto been regarded as a special category of UTI. However according to the new classification of UTI, recurrent UTI is now part of acute uncomplicated UTI together with the sporadic episodic UTI, ${ }^{7,8}$ provided the individuals have no structural or functional urinary tract abnormalities. It is defined as more than two separate episodes of uncomplicated UTI in the preceding six months or at least three episodes in the preceding twelve months, confirmed by bacteriological culture. ${ }^{54-56}$ They are symptomatic UTIs that occur after resolution of an earlier episode usually following appropriate treatment, and include relapses (i.e. recurrent UTIs caused by previously isolated bacteria after treatment and with a negative urine culture in the intervening period) or a recurrent UTI caused by a different bacteria from the one isolated in the previous episode. ${ }^{57}$

Recurrent UTIs are quite common and are a cause of significant morbidities to the individual woman as well as impact on her social and sexual well-being due to the recurrence of symptoms. Of the women who get one episode of acute uncomplicated UTI, $25-50 \%$ will experience recurrent UTI, ${ }^{9,13,56}$ which is a significant proportion and number considering the proportion who get at least one episode. 
Most recurrent UTIs are thought to represent infection with the same microorganism. ${ }^{57,58}$ These are most commonly seen in young sexually active women, and the major route being ascendance, ${ }^{54}$ just like in the sporadic episodes. The same micro-organisms responsible for sporadic episodes of uncomplicated UTI are also responsible for recurrent UTIs. E. coli accounts for the highest proportion (70-95\%), and S. saprophyticus accounts for $10-15 \% .{ }^{23,59}$ Some of the explanations for the recurrences include a suggestion that following resolution of a UTI, small numbers of the original strains of uropathogens persist in the hosts urinary system e.g. as infection stones (i.e P. mirabilis), from which a new infection may arise. Infections may also arise from a reservoir of bacteria in the vagina and/or alimentary system of a susceptible individual. ${ }^{59}$

The risk factors or recurrent UTI are essentially the same as those responsible for sporadic episodes of UTI, suggesting possible individual susceptibility to UTI. They include behavioural factors such as frequency of sexual intercourse, new partnerships and use of diaphragm with spermicides. ${ }^{54,58}$ In a case-control study, Scholes et al. ${ }^{41}$ reported that women with recurrent urinary tract infections were ten times more likely to have had sexual intercourse more than nine times in the preceding twelve months and twice more likely to have used spermicides in the same period. They also had a higher number of sexual partners than those without recurrent UTI. These factors are said to increase the risk of vaginal and urethral colonisation by uropathogens $^{58}$ just like in the sporadic UTIs. A maternal history of UTI and history of UTI before age 15 was also shown to be associated with a risk of recurrent UTI, ${ }^{54}$ as well as family relatives harbouring the uropathogens, suggesting a possible genetic predisposition to UTI. ${ }^{60,61}$

Among biological/genetic factors known to increase the risk of recurrent UTI, are anatomical factors, i.e. short distance between the urethra and anus, ${ }^{35}$ the location of the urethral meatus relative to vaginal introitus and anus; and biochemical i.e. lower levels of 25 -hydroxyvitamin D. ${ }^{62}$ Non-secretors of histo-blood group antigens are said to be more susceptible to recurrent UTI, though some studies have not shown such an association. ${ }^{61}$ Interleukin-8 receptor (IL-8R) or CXCRI, which has been shown to be expressed to a significant lower extent in pyelonephritis-prone children and their relatives, ${ }^{63}$ is thought to play a part in the pathogenesis of recurrent UTI.

\section{Discussion}

Acute uncomplicated urinary tract infections (UTIs) are very common in sexually active healthy, premenopausal women., 3 Reportedly about half of women globally will have at least one episode in their life time and up to about half of that will have at least three episodes in a year. Thus the magnitude thereof in premenopusal women is significant. It has major social and health impact as well as affects the quality of life of these individuals, and is associated with high health costs due to its prevalenc and need for investigative procedures and repeated treatment for the recurrences. ${ }^{64}$ Although uncomplicated UTI in premenopausal women is considered a relatively benign condition with no major long-term sequelae, it is nevertheless responsible for significant mordibity including effects on one's sexual functioning.

Genitourinary symptoms such as vaginal discharge, pruritus, pain during micturition, frequency and urgency are quite common complaints among the same group of women. Not all of these are attributable to urinary tract infections. Bent $\mathrm{S}$ et al. ${ }^{1}$ reported that the probability of women with dysuria, frequency or gross haematuria having UTI in a primary setting was $50 \%$, and that vaginal discharge and irritation reduce the probability thereof by $20 \%$. On the other hand, dysuria and frequency without vaginal discharge increase the probability of cystitis to $>90 \%,{ }^{2}$ still not hundred percent. These underscore the importance of confirmatory tests especially in cases of recurrences or when there is lack of antimicrobial response.

The pathogenesis of uncomplicated UTI, both sporadic and recurrent, has been subject for numerous studies essentially to try and understand why a healthy woman living within the community and with no urinary tract abnormalities gets UTI. Equally important has been the need to establish the main pathogenic organisms, their sources, how they get into the urinary tract and manage to survive and produce disease. It is said to be a complex process, which is influenced by the individual's genito-urinary and alimentary tracts' structural and physiological properties on the one hand, and the virulence properties of the infecting organisms on the other. ${ }^{14,15}$ The local innate immune mechanisms of the vagina, whose colonisation by bacteria is thought to be a critical initial step in the pathogenesis of UTI, and that of the urinary tract, have to be breached somehow for infection to occur. It is reported that in community-acquired UTI, the virulence properties of the infecting uropathogens are more important than the individual's biological and behavioural factors. This is perhaps more critical in recurrent UTI where the infecting microorganisms can survive in the vagina, alimentary or urinary tract, leading to re-infection in susceptible individuals. ${ }^{55,60} E$. coli being the commonest microorganism causing UTI in these women, has been shown to display critical virulence properties, which enable it to not only cause the initial infection but subsequent ones as well, as it is able to survive in reservoir forms in the vagina and alimentary tracts from where it re-infects the urinary tract. There is perhaps need for more studies to understand the real origin or source of UPEC, which has been thought to originate from contaminated poultry products, ${ }^{28}$ as that would facilitate preventive strategies.

The fact that a previous infection is a risk factor for another episode, and that recurrent UTI is a risk factor for a new episode of UTI, underline the fact that factors which might have led to the initial infectious episode may still be persisting. It calls for studies to better understanding the risk factors and their operational mechanisms. It also necessitates thorough treatment, followed by education and counselling on preventive strategies, after one episode or even before, as a part of general reproductive health education. The possibility of a male partner infecting his female partner from infections such as chronic prostatitis ${ }^{19,44}$ needs to be considered and investigated especially in recurrent UTI. It also underscores the importance of investigating ones sexual partner in case of recurrent UTI.

Penile-vaginal intercourse, with or without barrier contraceptive methods, namely diaphragm, and male condoms, with or without spermicides and spermicides themselves are major behavioural and potentially avoidable risk factors. ${ }^{15,37,41}$ Since vaginal introital and urethral meatal trauma during sexual intercourse is considered possible mechanisms through which sexual intercourse facilitates vaginal colonisation by uropathogens and their entrance into the urinary bladder, it is not surprising that community-acquired UTI occurs at the peak of a woman's sexual life. Young people are not only creative but also expolorative and enquisitive in all aspects of life including sexual activities. HAI, which is reportedly quite common amongst them globally, ${ }^{45,46}$ has been reported to be a risk factor thereto. ${ }^{40,45,46}$ Quite often these same individuals engage in other forms of sexual activities such as digital stimulation, receptive oral sex which may be annilingus or felatio or combinations thereof, which may also increase the risk of UTI in the female partners, as reported by Foxman et al. ${ }^{37}$ There is also an increase, though undocumented, in the use of sex toys such as vibrators and dildos by women either in lesbian 
relationships which are also on the rise among high school, college and university girls or solo. Whether, and to what extent these may increase or contribute to the increased risk of community-acquired UTI has not been determined. It is however worth mentioning and possible research subject.

Other common sexual practice which have not been investigated to elucidate their prevalence and/or impact on the individual's health, are dry and wet sex. These are widely practiced in some parts of sub-Saharan Africa, by traditional societies. Dry sex is common in countries in Central Africa and the Caribbeans. ${ }^{65,66}$ The process of drying the vagina following arousal or during sexual intercourse include insertion of dry leaves, powder, dry cloths, douching which on their own may bruise the vaginal walls. Dry sex is known to cause trauma to the vaginal walls. ${ }^{65,67,68}$ All these practices may increase the risk of infection i.e. colonisation of the vagina which may then be pushed into the urethra and bladder leading to UTI. The mechanism through which wet sex, which is practiced and preferred by some communities or individuals, may increase risk of UTI has not been documented. It may facilitate the movement of bacteria from the anus into the vagina and urethral meatus, because of the excessive wetness, or the process required to increase the woman's lubrication, requiring prolonged stimulation of the woman digitally or rubbing the penis against the clitoris and labia, which may cause trauma. It is an area which may require research studies to establish an association if any and possible mechanisms. Such information would be invaluable in informing preventive strategies.

The international health community and related agencies have expended significant resources on advocating for safer sex, including delaying sexual debut, having few sexual partners and one at a time, use of barrier contraceptive methods such as male or female condoms to prevent unwanted pregnancy, STIs, HIV and HPV responsible for cancer of cervix, which have led to an increase in the uptake of condoms and in particular spermicide-coated ones, by young people. The fact that using of spermicide-coated condoms may increase the risk of community-acquired UTI in young sexually women, may compel them to stop using condoms, which will undoubtedly erode the gains made so far in this regard.

There is therefore need for paradigm shift in sexual and reproductive health programming at the international as well as national levels; including packaging of health messages that appropriate and relevant for certain groups of individuals; service delivery, in particular enquiring into sexual practices during history taking in cases of genitourinary complaints. Health workers need to provide appropriate health education and counselling to young women at every contact on the factosr increasing risk of UTI, and possible preventive strategies. They should also ensure proper clinical diagnsosis and adequate treatment of UTI. The foregoing calls for appropriate and locally/ nationally relevant operations researches to understand the magnitude and determinants of uncomplicated UTI, thus informing programming and health care delivery and messaging.

\section{Acknowledgements}

None.

\section{Conflicts of interest}

None.

\section{References}

1. Nicolle LE. Epidemiology of urinary tract infections. Infect Med. 2001;18:153-162.
2. Foxman B. Epidemiology of urinary tract infections: incidence, morbidity, and economic costs. Am J Med. 2002;113(Suppl 1A):5S-13S

3. Hooton TM, Scholes D, Hughes JP, et al. A prospective study of risk factors for symptomatic urinary tract infection in young women. $N$ Engl J Med. 1996;335(7):468-474.

4. Schappert SM, Rechtsteiner EA. Ambulatory medical care utilization estimates for 2007. Vital Health Stat. 2011;13(169):1-38

5. Nicolle LE, Friesen D, Harding GK, et al. Hospitalization for acute pyelonephritis in Manitoba, Canada, during the period from 1989 to 1992; impact of diabetes, pregnancy, and aboriginal origin. Clin Infect Dis. 1996;22(6):1051-1056.

6. Pinson AG, Philbrick JT, Lindbeck GH, et al. ED management of acute pyelonephritis in women: A cohort study. Am J Emerg Med. 1994;12(3):271-278.

7. Simelov V, Naber K, Johansen TEB. Improved classification of urinary tract infection: Future considerations. Eur Urol Suppl. 2016;15(4):7180 .

8. Grabe M, Bartoletti R, Bjerklund Johansen TE, et al. guidelines on urological infections. European Association of Urology. 2015.

9. Foxman B, Gillespie B, Koopman J, et al. Risk factors for second urinary tract infection among college women. Am J Epidemiol. 2000;151(12):1194-1205.

10. Foxman B, Barlow R, D Arcy H, et al. Urinary tract infection: selfreported incidence and associated costs. Ann Epidemiol. 2000;10(8):509515

11. Fihn SD. Acute Uncomplicated Urinary Tract Infection in Women. $N$ Engl J Med. 2003;349:259-266.

12. Griebling TL. Urologic diseases in America project trends in resource use for urinary tract infections in women. J Urol. 2005;173(4):12811287.

13. Foxman B. Recurring urinary tract infection: incidence and risk factors. Am J Public Health. 1990;80(3):331-333.

14. Hooton TM. Pathogenesis of urinary tract infections: an update. $J$ Antimicrob Chemother. 2000;46Suppl1:1-7.

15. Fihn SD, Boyko EJ, Normand EH, et al. Association between use of spermicide-coated condoms and Escherichia coli urinary tract infection in young women. Am J Epidemiol. 1996;144(5):512-520.

16. Kunin CM. Urinary tract infections. Detection, prevention, and management. ( $5^{\text {th }}$ edn), Williams \& Witkins, USA. 1997.

17. Sobel JD. Urinary tract infections. In: Mandell GL, Douglas RG, Bennett JE, Dolin R, (Eds.), Mandell, Douglas, and Bennett's Principles and Practice of Infectious Diseases. ( $7^{\text {th }}$ edn), Elsevier Churchill Livingstone, Philadelphia, USA. 2009;pp.957-985.

18. Russo TA, Stapleton A, Wenderoth S, et al. Chromosomal restriction fragment length polymorphism analysis of Escherichia coli strains causing recurrent urinary tract infections in young women. $J$ Infect Dis. 1995;172(2):440-445.

19. Foxman B. Epidemiology of urinary tract infections: incidence, morbidity, and economic costs. Am JMed. 2002;113(Suppl 1A):5S-13S.

20. Johnson JR. Virulence factors in Escherichia coli urinary tract infection. Clin Microbiol Rev. 1991;4(1):80-128.

21. Donnenberg MS, Welch R. Virulence determinants of uropathogenic Escherichia coli. In: Mobley HLT \& Warren JW (Eds.), Urinary Tract Infections: Molecular Pathogenesis and Clinical Management, American Society for Microbiology, Washington, USA. 1996;pp.135-174.

22. Mulvey MA. Adhesion and entry of uropathogenic Escherichia coli. Cell Microbiol. 2002;4(5):257-271. 
23. Ronal A. The etiology of urinary tract infection: traditional and emerging pathogens. Dis Mon. 2003;49(2):71-82.

24. Farooqi BJ, Shareeq F, Rizvi QK, et al. Changing pattern of antimicrobial susceptibility of organisms causing community acquired urinary tract infections. J Pak Med Assoc. 2000;50(11):369-373.

25. Svanborg C, Godaly G. Bacterial virulence in urinary tract infection Infect Dis Clin North Am. 1997;11(3):513-529.

26. Alteri CJ, Mobley HLT. Escherichia coli Physiology and Metabolism Dictates Adaptation to Diverse Host Microenvironments Curr Opin Microbiol. 2011;15(1):3-9.

27. Johnson JR, Russo TA. Molecular epidemiology of extra intestina pathogenic (uropathogenic) Escherichia coli. Int J Med Microbiol 2005;295(6-7):383-404.

28. Rodriguez-Siek KE, Giddings CW, Doetkott C, et al. Comparison of Escherichia coliisolates implicated in human urinary tract infection and avian colibacillosis. Microbiology. 2005;151(Pt 6):2097-2110.

29. Chen SL, Hung CS, Pinkner JS, et al. Positive selection identifies an in vivo role for FimH during urinary tract infection in addition to mannose binding. Proc Natl Acad Sci U S A. 2009;106(52):22439-22444.

30. Alteri CJ, Smith SN, Mobley HL. Fitness of Escherichia coli during urinary tract infection requires gluconeogenesis and the TCA cycle. PLoS Pathog. 2009;5(5):e1000448.

31. Roesch PL, Redford P, Batchelet S, et al. Uropathogenic Escherichia coli use d-serine deaminase to modulate infection of the murine urinary tract. Mol Microbiol. 2003;49(1):55-67.

32. Wiles TJ, Kulesus RR, Mulvey MA. Origins and Virulence Mechanisms of Uropathogenic Escherichia Coli. Exp Mol Pathol. 2008;85(1):11-19.

33. Davis JM, Carvalho HM, Rasmussen SB, et al. Cytotoxic necrotizing factor type 1 delivered by outer membrane vesicles of uropathogenic Escherichia coli attenuates polymorphonuclear leukocyte antimicrobial activity and chemotaxis. Infect Immun. 2006;74(8):4401-4408.

34. Kuroda M, Yamashita A, Hirakawa H, et al. Whole genome sequence of Staphylococcus saprophyticus reveals the pathogenesis of uncomplicated urinary tract infection. Proc Natl Acad Sci U S A. 2005;102(37):13272 13277.

35. Hooton TM, Stapleton AE, Roberts PL, et al. Perineal anatomy and urine-voiding characteristics of young women with and withou recurrent urinary tract infections. Clin Infect Dis. 1999;29(6):1600 1601 .

36. Mulvey MA, Schilling JD, Hultgren SJ. Establishment of a Persistent Escherichia coli Reservoir during the Acute Phase of a Bladder Infection. Infect Immun. 2001;67(7):4572-4579.

37. Foxman B, Geiger AM, Palin K, et al. First time urinary tract infection and sexual behaviour. Epidemiology. 1995;6(2):162-168

38. Moore EE, Hawes SE, Scholes D, et al. Sexual intercourse and risk of symptomatic urinary tract infection in post-menopausal women. J Gen Intern Med. 2008;23(5):595-599.

39. Lach PA, Elster AB, Roghmann KJ. Sexual behavior and urinary tract infection. Nurse Pract. 1980;5(1):27-28.

40. Handley MA, Reingold AL, Shiboski S, et al. Incidence of acute urinary tract infection in young women and use of male condoms with and without nonoxynol-9 spermicides. Epidemiology. 2002;13(4):431-436.

41. Scholes D, Hooton TM, Roberts PL, et al. Risk Factors for Recurrent Urinary Tract Infection in Young Women. J Infect Dis. 2000;182(4):1177-1182

42. Nicolle LE, Harding GK, Preiksaitis J, et al. The association of urinary tract infection with sexual intercourse. J Infect Dis. 1982;146(5):579583.
43. Strom BL, Collins M, West SL, et al. Sexual activity, contraceptive use, and other risk factors for symptomatic and asymptomatic bacteriuria: a case-control study. Ann Intern Med. 1987;107(6):816-823.

44. Al-Wali W, Hamilton-Miller JM, Joshi S, et al. A case of recurrently sexually transmitted urinary tract infection. Genitourin Med. 1989;65(6):397-398.

45. Coull N, Mastoroudes H, Popert R, et al. Redefining urological history taking - anal intercourse as the cause of unexplained symptoms in heterosexuals. Ann R Coll Surg Engl. 2008;90(5):403-405.

46. Lema VM. Urinary tract infection in young healthy women following heterosexual anal intercourse: Case reports. Afr $J$ Reprod Health. 2015;19(2):133-138.

47. Foxman B, Frerichs RR. Epidemiology of urinary tract infection: I. Diaphragm use and sexual intercourse. Am J Public Health. 1985;75(11):1308-1313

48. Foxman B, Marsh J, Gillespie B, et al. Condom use and first-time urinary tract infection. Epidemiology. 1997;8(6):637-641.

49. Fihn SD, Boyko EJ, Chen CL, et al. Use of spermicide-coated condoms and other risk factors for urinary tract infection caused by Staphylococcus saprophyticus. Arch Intern Med. 1998;158(3):281-287.

50. Rosenstein IJ, Stafford MK, Kitchen VS, et al. Effect on normal vaginal flora of three intravaginal microbicidal agents potentially active agains human immunodeficiency virus type 1. J Infect Dis. 1998;177(5):13861390 .

51. Hooton TM, Hillier S, Johnson C, et al. Escherichia coli bacteriuria and contraceptive method. JAMA. 1991;265(1):64-69.

52. Herthelius-Elman M, Möllby R, Nord CE, et al. The effect of amoxicillin on vaginal colonisation resistance and normal vaginal flora in monkeys. J Antimicrobial Chem. 1992;29(3):329-340.

53. Smith HS, Hughes JP, Hooton TM, et al. Antecedent antimicrobial use increases the risk of uncomplicated cystitis in young women. Clin Infect Dis. 1997;25(1):63-68.

54. Epp A, Larochelle A, Lovatsis D, et al. Recurrent urinary tract infection. J Obstet Gynaecol Can. 2010;32(11):1082-1101.

55. Nosseir SB, Lind LR, Winkler HA. Recurrent uncomplicated urinary tract infections in women: a review. $J$ Womens Health (Larchmt). 2012;21(3):347-354

56. Aydin A, Ahmed K, Zaman I, et al. Recurrent urinary tract infections in women. Int Urogynecol J. 2015;26(6):795-804.

57. Hooton TM Gupta K. Recurrent urinary tract infection in women. 2014

58. Franco AV. Recurrent urinary tract infections. Best Pract Res Clin Obstet Gynaecol. 2005;19(6):861-873.

59. Nosseir SB, Lind LR, Winkler HA. Recurrent uncomplicated urinary tract infections in women: a review. $J$ Womens Health (Larchmt). 2012;21(3):347-354.

60. Johnson JR, Clabots C. Sharing of virulent Escherichia coli clones among household members of a woman with acute cystitis. Clin Infect Dis. 2006;43(10):e101-e108

61. Zaffanello M, Malerba G, Cataldi L, et al. Genetic risk for recurrent urinary tract infections in humans: a systematic review. J Biomed Biotechnol. 2010;2010:321082.

62. Nseir W, Taha M, Nemarny H, et al. The association between serum levels of vitamin D and recurrent urinary tract infections in premenopausal women. Int J Infect Dis. 2013;17(12):1121-1124

63. Lundstedt AC, Leijonhufvud I, Ragnarsdottir B, et al. Inherited susceptibility to acute pyelonephritis: a family study of urinary tract infection. J Infect Dis. 2007;195(8):1227-1234. 
64. Russo TA, Johnson JR. Medical and economic impact of extra intestinal infections due to Escherichia coli: focus on an increasingly important endemic problem. Microbes Infect. 2003;5(5):449-456.

65. Nyirenda MJ. A study of the behavioural aspects of dry sex practice in urban Lusaka. Eighth International Conference on AIDS. 1992

66. Brown JE, Brown RC. Traditional intravaginal practices and the heterosexual transmission of disease. Sex Transm Dis. 2000;27(4):183187.
67. Kilmarx PH, Limpakarnjanarat K, Supawitkul S, et al. Mucosal disruption due to use of a widely distributed commercial vaginal product: potential to facilitate HIV transmission. AIDS. 1998;12(7):767-773.

68. Brown JE, Ayowa OB, Brown RC. Dry and tight: sexual practices and potential AID S risk in Zaire. Soc Sci Med. 1993;37(8):989-994. 